Revue internationale P.M.E.

Économie et gestion de la petite et moyenne entreprise

\title{
Les modèles intégrés de la décision d'exporter en PME/PMI : synthèse des recherches depuis 20 ans
}

\section{Marc Gibiat}

Volume 7, numéro 2, 1994

URI : https://id.erudit.org/iderudit/1008387ar

DOI : https://doi.org/10.7202/1008387ar

Aller au sommaire du numéro

Éditeur(s)

Presses de l’Université du Québec

ISSN

0776-5436 (imprimé)

1918-9699 (numérique)

Découvrir la revue

Citer cet article

Gibiat, M. (1994). Les modèles intégrés de la décision d'exporter en PME/PMI : synthèse des recherches depuis 20 ans. Revue internationale P.M.E., 7(2), 11-29. https://doi.org/10.7202/1008387ar

\section{Résumé de l'article}

L'objectif de cet article est d'établir une synthèse des recherches portant sur les modèles intégrés de la décision d'exporter. Nous soulignerons les composantes principales de ces modèles (le niveau incitatif: stimuli et variables exogènes; le niveau décisionnel : la prise de conscience, l'intérêt, l'intention, l'essai, la décision; le niveau comportemental : l'attitude managériale), pour ensuite dégager les constantes et les points de divergences. Enfin, nous mettrons en valeur les implications managériales qui découlent de ces études, avant de conclure en présentant les voies de recherche à travailler. 


\title{
Les modèles intégrés de la décision d'exporter en PME/PMI : synthèse des recherches depuis 20 ans
}

Marc GIBIAT*

ESC Chambéry

\begin{abstract}
RÉSUMÉ
L'objectif de cet article est d'établir une synthèse des recherches portant sur les modèles intégrés de la décision d'exporter. Nous soulignerons les composantes principales de ces modèles (le niveau incitatif: stimuli et variables exogènes; le niveau décisionnel: la prise de conscience, l'intérêt, l'intention, l'essai, la décision; le niveau comportemental: l'attitude managériale), pour ensuite dégager les constantes et les points de divergences. Enfin, nous mettrons en valeur les implications managériales qui découlent de ces études, avant de conclure en présentant les voies de recherche à travailler.
\end{abstract}

\begin{abstract}
The aim of this article is to establish a summary of research concerning integrated models of decision to export. The main elements of these models will be emphasised (the level of motivation: stimuli and exogenous variables; the level of decision-making: the awareness, the interest, the intention, the trial, the decision; the behavioural level: the managerial attitude), before identifying common and differing characteristics. Finally, the managerial implications which result from studies will be presented, concluding by recommanding research paths to be followed.
\end{abstract}

* Marc Gibiat possède un diplôme de DEA en sciences de gestion (École supérieure des affaires de Grenoble); il est de plus diplômé de l'Institut d'études politiques de Grenoble et possède une maîtrise en sciences économiques (Université Pierre Mendès-France de Grenoble). Il est présentement enseignant-chercheur à l'École supérieure de commerce de Chambéry et poursuit un doctorat en sciences de gestion à l'École supérieure des affaires, Université Pierre Mendès-France. Le thème de sa thèse est «L'étude du comportement des PME/PMI à l'international». Adresse: Enseignant-chercheur, École supérieure de commerce de Chambéry, B.P. 9451, 73094 Chambéry, France. 


\section{RESUMEN}

El objectivo de este artículo es establecer una síntesis de las investigaciones llevadas a cabo sobre los modelos integrados de la decisión de exportar. Estudiaremos los componentes principales de estos modelos (el nivel incitativo: estímulos y variables exógenas; el nivel decisional: la toma de conciencia, el interés, la intención, la prueba, la decisión; el nivel de comportamiento: la actitud gestionaria), para poder en consecuencia aislar las constantes y los puntos de divergencias. Por último, destacaremos las implicaciones en la gestión que derivan de estos estudios, antes de concluir presentando los ejes de investigación a desarrollar.

\section{Introduction}

Au cours des vingt dernières années, de nombreuses études sur l'expansion internationale des PME/PMI ont été réalisées. Ainsi, Bilkey (1978) a relevé dans sa revue de la littérature portant sur dix années, 46 études sur le comportement à l'exportation des PME/PMI. Miesenbock (1988), pour sa part, recense 139 articles dans sa revue de la littérature concernant l'exportation et les PME. Dans un même ordre d'idée, Aaby et Slater (1989) ont comptabilisé sur la période 1978-1988 près de 55 études ayant comme champ d'analyse l'influence des caractéristiques managériales sur la performance à l'exportation des PME/PMI.

La plupart des études portant sur l'expansion internationale des PME cherchent à mettre en valeur les déterminants de l'exportation. Une classification de ces déterminants peut prendre la forme suivante (Dichtl, Leibold, Köglmayr et Müller, 1984):

- le marché national : intensité de la concurrence, taille et niveau technologique du marché, comportement du consommateur, programme de promotion des exportations, etc.;

- les marchés étrangers : intensité de la concurrence, comportement du consommateur, risque pays, barrières à l'exportation, etc. ;

- l'entreprise: structure organisationnelle, nombre d'employés, part de marché, capacité de production, etc.;

- le dirigeant : expérience à l'étranger, compétence en langues étrangères, attitude envers le risque, propension à innover, nombre de personnes concernées par la prise de décision à l'exportation.

L'accumulation de telles listes de facteurs, en dépit de leur mérite d'avoir été révélées, ne suffit pas, par exemple, à identifier les caractéristiques distinctives des entreprises non exportatrices qui pourtant semblent (comme les autres) pouvoir réussir à l'exportation. 
C'est pourquoi certains auteurs ont axé leurs réflexions au-delà de la simple analyse des déterminants du comportement exportateur des PME/PMI. Pour ce faire, ils ont intégré ces facteurs explicatifs à un processus de décision dans lequel s'engagent les entreprises: on parle alors de «modèles intégrés de la décision d'exporter». Ces modèles peuvent être considérés comme des processus qui, à la suite d'acquisition et d'intégration d'informations sur les marchés étrangers, entraînent un engagement progressif et croissant de l'entreprise au niveau international (Johanson et Vahlne, 1977). Ces auteurs avancent que cet engagement à l'international s'explique par un processus incrémental d'ajustements à l'environnement et à la firme elle-même, plus que par l'adoption d'une stratégie délibérée. L'absence d'expérience, le manque d'informations appropriées et l'incertitude qui règne sur les marchés étrangers sont les fondements explicatifs de ce processus incrémental.

À l'origine, ces modèles intégrés de la décision d'exporter se basent sur le processus d'adoption d'une innovation (Rogers, 1962). Ce processus est initialisé par un ensemble de facteurs qui conduisent le décideur à reconnaître une opportunité en termes d'innovation. L'accumulation d'informations et la mise en place d'une évaluation détermine le choix final du décideur en faveur (ou non) de l'adoption d'une innovation. Simmonds et Smith (1968) ont repris ce cadre d'analyse pour étudier le processus de décision à l'exportation.

Deux articles synthétiques (Bilkey,1978 et Roux, 1986) ont présenté les études réalisées sur les modèles intégrés de la décision d'exporter. Nous nous proposons de reprendre ces travaux en les complétant avec des modèles non cités ou plus récents. L'objectif de cet article est de mettre en lumière les composantes de ces modèles, de souligner les constantes et les points de divergences et, enfin, de présenter les implications managériales.

\section{Les modèles intégrés de la décision d'exporter en PME/PMI : cadre conceptuel et présentation générale}

Ces modèles (comme la quasi-totalité des travaux concernant le comportement stratégique des PME/PMI) placent le dirigeant au centre de l'analyse. On est donc ici dans une optique «behaviorale» qui se préoccupe des comportements individuels et collectifs dans la genèse des décisions au sein de l'organisation. Ce contexte théorique sous-entend l'hypothèse suivante: les décisions stratégiques d'une PME sont directement liées à la formation, à l'expérience, aux valeurs et aux convictions de son dirigeant.

Dans la plupart des petites entreprises, on est très souvent en présence d'un unique décideur qui s'engage seul dans la démarche stratégique (Olson et Wiedersheim-Paul, 1978). Il est donc reconnu qu'un individu peut dominer 
le processus de décision. En outre, s'il existe plusieurs acteurs dans la prise de décision, il sera toujours fait référence à la personne qui coordonne la prise de décision.

Dans ce cadre, les modèles de la décision d'exporter en PME/PMI s'attachent:

- à analyser le rôle du dirigeant-propriétaire lors des différentes étapes du processus de décision à l'exportation;

- à souligner et à mesurer l'influence des caractéristiques de l'entreprise, de l'environnement et du dirigeant sur ce même processus de décision.

Outre l'atteinte de ces objectifs, les modèles doivent respecter certaines règles. Ainsi, pour Ditch, Köglmayr, Leibold et Müller (1984), un «bon modèle de décision» doit être:

- complexe: il incorpore un grand nombre de variables;

- explicatif: il ne se limite pas à la description des relations existantes, il les explique;

- dynamique: il suit la décision dans le temps;

- micro-économique: il examine la prise de décision au regard des acteurs économiques.

De façon plus précise, si l'on tente à partir des modèles que nous avons recensés ${ }^{1}$ de mettre en valeur leurs composantes communes, nous pouvons dresser le modèle type (figure 1).

Pour bien comprendre les différentes phases de ce modèle et la façon dont elles s'enchaînent, rappelons que le dirigeant joue un rôle central. En effet, il est dès la première phase (que l'on peut qualifier de phase «incitative») exposé à un certain nombre de «stimuli». Ces stimuli sont en mesure d'attirer l'attention du dirigeant sur l'existence de l'opportunité d'exporter.

Ces stimuli sont perçus (ou non) en fonction de l'influence de variables dites exogènes (explicatives). C'est au cours de cette étape appelée «processus interne» que le dirigeant filtre les stimuli en fonction des variables exogènes. Cette opération de filtre engage (ou non) le dirigeant dans le processus

1. Pavord et Bogart, 1975 ; Bilkey et Tesar, 1977; Wiedersheim-Paul, Olson et Welch, 1978; McConnel, 1979; Roux, 1979; Reid, 1980 ; Steinmann, Kumar et Wasner, 1980 ; Cavusgil et Nevin, 1981 ; Garnier, 1982 ; Welch, 1983, Ditch et al., 1984 ; Mescon et Vozikis, 1985 ; Jaffe, Nebenzahl et Pasternak, 1988 ; Sharma, 1988; Axinn, 1989 ; Higler et Hoover, 1990 ; Ortiz-Buonafina, 1990; Lim, Sharkey et Kim, 1991 ; Minor, Wu et Choi, 1991 ; Rao et Nairu, 1992; Czinkota et Ronkainen, 1993. 


\section{FIGURE 1}

Schéma de synthèse des modèles intégrés de la décision d'exporter en PME/PMI

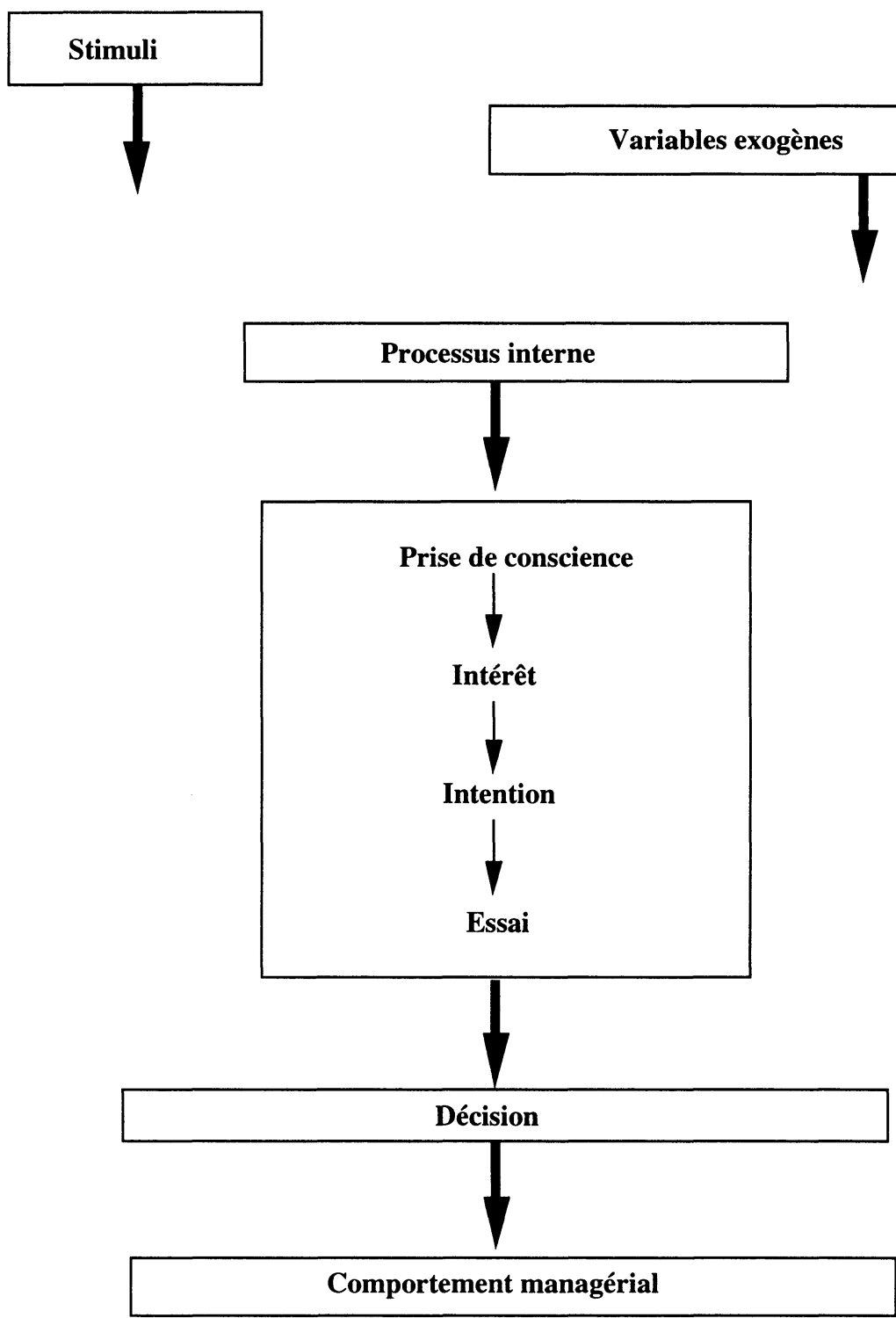


séquentiel de décision en lui-même, processus qui le conduira (ou non) à la décision d'exporter. Au-delà de la décision, on effectue une typologie du comportement managérial.

Le cadre général du modèle étant fixé, nous allons maintenant présenter successivement les stimuli, les variables exogènes, le processus interne et, enfin, les différentes étapes du processus de décision.

\section{Les stimuli ou variables perçues}

Les stimuli sont les éléments les plus dynamiques du modèle. Ils sont appelés aussi «facteurs attirant l'attention du dirigeant ${ }^{2}$ », c'est-à-dire des facteurs qui conduisent le dirigeant à reconnaître dans l'exportation une opportunité. Néanmoins, il faut noter qu'ils ne produisent un effet que dans la mesure où ils sont perçus : parfois ils sont donc latents, mais ce n'est que sous leur forme active qu'ils sont intégrés dans les différents modèles.

Les auteurs différencient généralement les stimuli externes des stimuli internes (tableau 1):

Les stimuli externes. Ils sont liés à l'environnement de l'entreprise et sont nombreux (la pression concurrentielle, les aides et incitations des pouvoirs publics, l'intégration économique...); mais le stimulus le plus souvent cité est la commande étrangère non sollicitée.

L'une des premières études sur l'influence des commandes étrangères non sollicitées a été réalisée par Simpson et Kujawa (1974). Leur étude porte sur un échantillon de $120 \mathrm{PME}$ dont 50 sont exportatrices et 70 non exportatrices. Pour $82 \%$ des sociétés exportatrices, le stimulus externe le plus important est la commande étrangère non sollicitée (dans une étude sur 35 entreprises Lee et Brasch (1978) remarquent que 24 d'entre elles ont répondu à une offre étrangère). Cependant, ils soulignent que $30 \%$ des entreprises non exportatrices ont été également sollicitées par des commandes étrangères, mais sans pour autant attirer leur attention sur l'opportunité d'exporter. Cela peut s'expliquer par le fait que d'autres facteurs jouent sur l'attention d'exporter via les commandes étrangères non sollicitées.

Ce fait est confirmé par le modèle de Reid (1981). Il note que les aides et les informations des agents publics stimulent les entreprises dans la reconnaissance de l'opportunité d'exporter, les amenant à penser que cela peut leur permettre de répondre plus rapidement et plus efficacement aux offres provenant de l'étranger.

2. Attention-evoking factors, Wiedersheim-Paul, Olson et Welch, 1978. 


\begin{tabular}{|c|c|c|c|c|c|c|c|c|c|c|c|}
\hline & 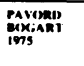 & 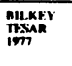 & 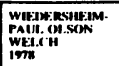 & 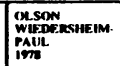 & MCONNEL & poux & 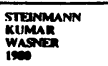 & 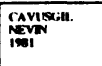 & ision & GANuES & WELCH \\
\hline \multicolumn{12}{|l|}{ STIMUIII INTERNES } \\
\hline \multirow{2}{*}{\multicolumn{12}{|c|}{$\begin{array}{l}\text { Carkekeristiques uniques dut } \\
\text { provuli }\end{array}$}} \\
\hline & & & & & & & & & & & \\
\hline Taille du marche interieur & * & & * & * & * & & & & & & * \\
\hline Objectifs de croissance & & & * & * & & & & * & * & & \\
\hline Nonihre d'employess & & * & & & & & * & & & * & \\
\hline \multirow{2}{*}{\multicolumn{12}{|c|}{$\begin{array}{l}\text { Forces de l'entreprise } \\
\text { STIMUI.I EXTERNES }\end{array}$}} \\
\hline & & & & & & & & & & & \\
\hline Intensite concurrentielle & & & * & * & & * & & & * & * & * \\
\hline Secteur d'activité & & & & & & & & * & & * & * \\
\hline Commandes étrangìres & * & * & * & * & & & * & & * & * & * \\
\hline $\begin{array}{l}\text { Saturation du march } \hat{\varepsilon} \\
\end{array}$ & & & & & * & ${ }^{*}$ & & & & & * \\
\hline Mesure gouvernementale & & & * & & & & & & & & \\
\hline \multicolumn{7}{|l|}{ Integration Economique } & & & & & \\
\hline \multicolumn{12}{|l|}{$\begin{array}{l}\begin{array}{l}\text { Potemticl des marchés } \\
\text { ctrangers }\end{array} \\
\end{array}$} \\
\hline & 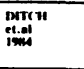 & 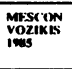 & $\operatorname{sinaman}$ & 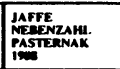 & $\operatorname{Nom}_{i=0}$ & 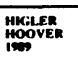 & 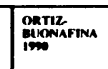 & 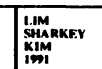 & Minon & mon & $\begin{array}{l}\text { ZZNKOTA } \\
\text { RONANANENE } \\
\text { imS }\end{array}$ \\
\hline \multicolumn{12}{|l|}{ STIMULI INTERNES } \\
\hline \multicolumn{12}{|l|}{$\begin{array}{l}\text { Caracteristiques uniques } \\
\text { du produit }\end{array}$} \\
\hline Capacites de l'entreprise & & * & * & * & & & $*$ & * & * & * & * \\
\hline Taille du marché intérieur & $*$ & & & & * & & & & * & * & * \\
\hline Objectifs de croissance & & & & & * & * & & & * & & * \\
\hline Nombre d'employes & * & * & * & * & * & & * & & & * & * \\
\hline Forces de l'entreprise & & & * & * & & & & & $*$ & * & * \\
\hline \multicolumn{12}{|l|}{ STIMULI EXTERNES } \\
\hline Intensité concurrentielle & * & & * & & & * & & & * & * & * \\
\hline Secteur d'activite & * & & & & & & & & * & & * \\
\hline Commandes ćtrang̀res & * & ${ }^{*}$ & & & * & & * & * & * & & * \\
\hline Saturation du marché & & & & & & * & & & * & & * \\
\hline Mesure gouvernementale & & & * & & & & & & & & * \\
\hline \multicolumn{12}{|l|}{ Intégration économique } \\
\hline $\begin{array}{l}\begin{array}{l}\text { Polentiel des marchés } \\
\text { citrangers }\end{array} \\
\end{array}$ & & & * & * & & * & & * & * & & * \\
\hline
\end{tabular}

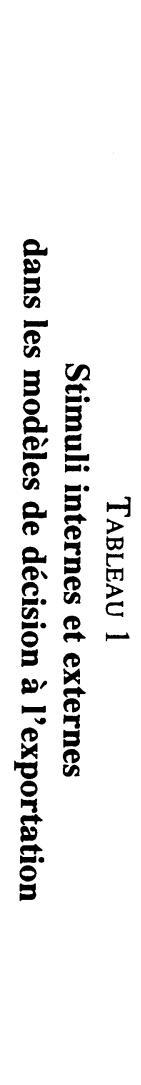


Les stimuli internes. Ils sont liés aux objectifs de croissance de la firme dans un environnement donné. Des perspectives de difficulté ou de réussite peuvent conduire à la création de stimuli favorisant l'exportation. Les stimuli internes les plus souvent cités sont: les caractéristiques uniques du produit, les capacités excédentaires de l'entreprise, la taille du marché intérieur, les objectifs de croissance, le nombre d'employés, les forces de l'entreprise.

Les capacités excédentaires (présentes ou à venir) de la firme sont les stimuli qui ont été révélés dès les premières études (Simpson et Kajuwa, 1974). Il est important de souligner que les capacités excédentaires ne sont pas uniquement reliées à la production. Elles concernent toutes les ressources de l'entreprise (finance, management, employés...).

Pour ce qui est de la compétence unique de la firme (ou caractéristiques uniques du produit), il faut souligner qu'elle est très attrayante pour les acheteurs étrangers, et ce, d'autant plus qu'ils y auront été sensibilisés auparavant (Wiedersheim-Paul, Olson et Welch, 1978).

\section{Les variables exogènes ou variables explicatives}

Avant de mettre en lumière les variables exogènes (ou explicatives), il est nécessaire de préciser le fait suivant: la quasi-totalité des PME sont exposées à des stimuli, mais une partie seulement de ces entreprises vont s'engager à l'exportation. Cela ne signifie pas que la présence de deux stimuli ou plus auraient plus d'effet sur une entreprise qu'un seul. Cela ne veut pas sous-entendre non plus que la combinaison de certains stimuli favoriserait plus que d'autres une réaction positive des entreprises par rapport à l'exportation. En d'autres termes, les stimuli ne peuvent expliquer à eux seuls l'engagement de la PME dans la voie de l'exportation. Seule la combinaison de stimuli et de variables explicatives peut déclencher le processus de décision.

On peut distinguer trois catégories de variables exogènes:

- les caractéristiques de l'environnement;

- les caractéristiques de l'entreprise;

- les caractéristiques du dirigeant.

Tout d'abord, il est nécessaire de préciser que des variables sont entendues comme variables exogènes, mais parfois également comme stimuli. C'est notamment le cas de l'existence d'une compétence unique (en termes de produit, par exemple). Cette variable est exogène dans le sens où elle peut expliquer l'engagement de l'entreprise à l'exportation. La compétence unique, et nous l'avons 
vu plus haut, est parfois considérée comme un stimulus si elle a attiré l'attention du dirigeant sur l'opportunité d'exporter. En définitive, le stimulus se différencie de la variable exogène par le fait («qu'il évoque un lien entre un événement donné et la possibilité d'exporter»: Welch, 1983)).

Des trois variables exogènes, nous avons choisi de faire ressortir les caractéristiques du dirigeant, car c'est la variable la plus déterminante. Les variables «environnement» (appartenance sectorielle, intensité de la concurrence...) et «entreprise» (taille, structure organisationnelle, caractéristiques du procédé de production...) sont moins déterminantes d'une part, et d'autre part, elles souffrent d'une proximité qui confère, par exemple, à l'appartenance sectorielle, le double statut de variable d'environnement (pour Garnier, 1982) et de variable d'entreprise (pour Cavusgil et Nevin, 1981).

Le récent modèle de Axinn (1989) reflète tout à fait le rôle déterminant du dirigeant. Cet auteur met en valeur une relation positive entre:

- une variable d'état, en l'occurrence, l'expérience du dirigeant en matière d'affaires internationales et son engagement à l'exportation ;

- des variables de perception du dirigeant et son engagement à l'exportation. En effet, le dirigeant perçoit le marché étranger comme un marché à plus fort potentiel de croissance que le marché local. Il pense que la compréhension de la documentation liée à l'exportation ne comporte pas de difficultés et, enfin, il passe outre la complexité que sous-entend l'expansion internationale. La façon dont il perçoit ces différentes variables détermine directement sa reconnaissance de l'occasion d'exporter.

L'influence de ces différentes variables est confirmée par d'autres auteurs (notamment Keng et Juan, 1989; Koh, 1990; Madsen, 1989). Simpson et Kujawa (1974) remarquent que la perception du coût, du profit ou encore du risque lié à l'exportation stimule la prise de décision, tandis que Olson et Wiedersheim-Paul (1978) mettent en valeur le style cognitif du dirigeant, c'est-à-dire la façon dont il récolte et traite les informations.

Il est intéressant de noter que Moon et Lee (1990) ne relèvent pas de relation significative entre les variables managériales et la reconnaissance de l'opportunité d'exporter. S'interrogeant sur cette conclusion, les deux auteurs soulignent que leur étude a été menée en Corée du Sud où les entreprises suivent à la lettre la recommandation du gouvernement: «exporter ou mourir». Ainsi, indépendamment de leurs caractéristiques, les dirigeants ont par obligation une orientation vers l'international. 
Pour faire une synthèse des caractéristiques du dirigeant comme variable exogène, on peut avancer la classification suivante (Roux, 1991):

- caractéristiques sociodémographiques : maîtrise des langues étrangères, niveau et type de formation, origine culturelle;

- caractéristiques psychographiques;

- les déterminants profonds et stables du comportement tels que la personnalité, les styles cognitifs, les motivations individuelles;

- les valeurs et attitudes individuelles qui sont des variables intermédiaires. On classe dans cette catégorie les attitudes vis-à-vis de l'exportation, l'orientation internationale, l'attitude envers le risque;

- les variables d'information qui traduisent le degré de connaissance du domaine et des procédures spécifiques à l'exportation, d'une part, et l'exposition aux médias, d'autre part.

\section{Le processus interne}

Il consiste en une opération de filtrage des informations perçues (stimuli) et facteurs explicatifs (variables exogènes). Le processus interne n'a pas fait l'objet de travaux approfondis soulignant le fait que cette phase est le plus souvent implicite au sein des modèles de décision d'exporter.

D'anciennes études sur la diffusion de l'innovation (Coleman, 1957) démontrent que l'amorce du processus de décision (donc le processus interne) est dépendant d'une succession d'événements non intentionnels. En d'autres termes, la reconnaissance de l'opportunité d'innover (ou d'exporter) se ferait de façon fortuite (ou incrémentale non consciente). Hassinger (1959) a critiqué cette position en affirmant que l'initialisation du processus de décision est active (et non passive), car déterminée par le comportement du décideur.

En définitive, on se demande si un besoin précède l'opportunité d'innover (ou d'exporter), ou si c'est la prise de conscience de l'intérêt de l'investissement de nouveaux marchés qui crée le besoin d'innover (ou d'exporter). Dans l'état actuel des recherches, il n'est pas possible de répondre à cette question.

\section{Les phases du processus de décision à l'exportation}

Le contenu et le nombre des phases peuvent varier d'un modèle à l'autre. Cependant, comme nous l'avons précisé plus haut, il est possible de déterminer un processus de décision générique qui comprend quatre phases. 
Phase 1 : perception de l'opportunité d'exporter («awareness» ou encore «exposure » selon Emery, 1962). Le décideur reconnaît qu'une stratégie d'exportation peut être considérée comme une bonne affaire. À ce stade, il souffre d'un manque total d'information au sujet de l'exportation. En outre, il n'est pas enclin à rechercher cette information. La fonction première de cette phase est d'amorcer le processus de décision en lui-même.

Phase 2 : intérêt pour l'exportation. Le dirigeant porte un vif intérêt pour cette stratégie. Le comportement du décideur est à partir de cette phase assurément intentionnel. Sa personnalité et ses valeurs affectent la recherche, la nature et le traitement des informations. Ce stade est appelé aussi stade de "l'information » (Beal et Bohlen, 1957) ou stade de la «connaissance» (Lavidge et Steiner, 1961).

Phase 3 : intention d'exporter. Le dirigeant adopte un comportement positif à l'égard de l'exportation. C'est certainement le stade le plus difficile à formaliser. En raison de sa position (entre le stade de l'intérêt et celui de l'essai), il est défini comme un essai mental. Le décideur applique mentalement à la situation présente et future sa volonté d'exporter. Toujours par un exercice mental, il tente d'évaluer les avantages et les inconvénients d'une telle décision. Des termes qui ont été avancés pour qualifier ce stade, c'est certainement la «conviction» (Roger et Yost, 1960) qui précise le mieux la nature de cette phase.

Phase 4 : essai. Le dirigeant intensifie notamment sa recherche d'informations sur les marchés étrangers (afin de pouvoir valider avec certitude leur essai) et évalue les capacités de son entreprise dans l'optique d'une stratégie d'exportation.

Le passage de la phase d'intérêt à la phase d'intention s'effectue par le biais d'une démarche cognitive, dans la mesure où les décideurs tentent par le recueil d'informations d'accroître leurs connaissances sur le thème qui les préoccupe : l'exportation. C'est, cette fois-ci, par une démarche «affective» que le dirigeant passe de la phase d'intérêt à la phase d'intention. Après avoir suscité l'intérêt du dirigeant, l'exportation devient un projet hypothétique.

La dernière démarche est comportementale : elle lie la phase d'intention à la phase d'essai. Le dirigeant adopte une attitude active en mesurant les chances de succès de son engagement à l'exportation. Après cette ultime phase, le dirigeant décide d'adopter (ou non) une stratégie d'exportation. Schématiquement, les quatre phases et les trois démarches prennent la forme suivante (figure 2).

Dans certains modèles, on note la présence d'une cinquième phase. En fait, elle dédouble la phase d'essai en une phase d'évaluation des capacités de l'entreprise à exporter et une phase d'essai à proprement parler, c'est-à-dire d'exportation expérimentale. 
Nous présentons au tableau 2, vingt-deux modèles de décision d'exporter. Pour chacun, on note les différentes phases du processus de décision ainsi que leur caractère formel ou informel. La dernière ligne du tableau (attitude managériale) correspond à l'existence d'une typologie du comportement du dirigeant qui a adopté une stratégie d'exportation. Par exemple, Bilkey et Tesar (1977), Roux (1979) et Cavusgil (1980) distinguent un engagement minimal d'un engagement délibéré. Par ailleurs, la distinction entre comportement actif, passif et réactif est faite par Wiedersheim-Paul, Olson et Welch (1978).

FIGURE 2

Les quatre phases du processus de décision d'exporter en PME/PMI

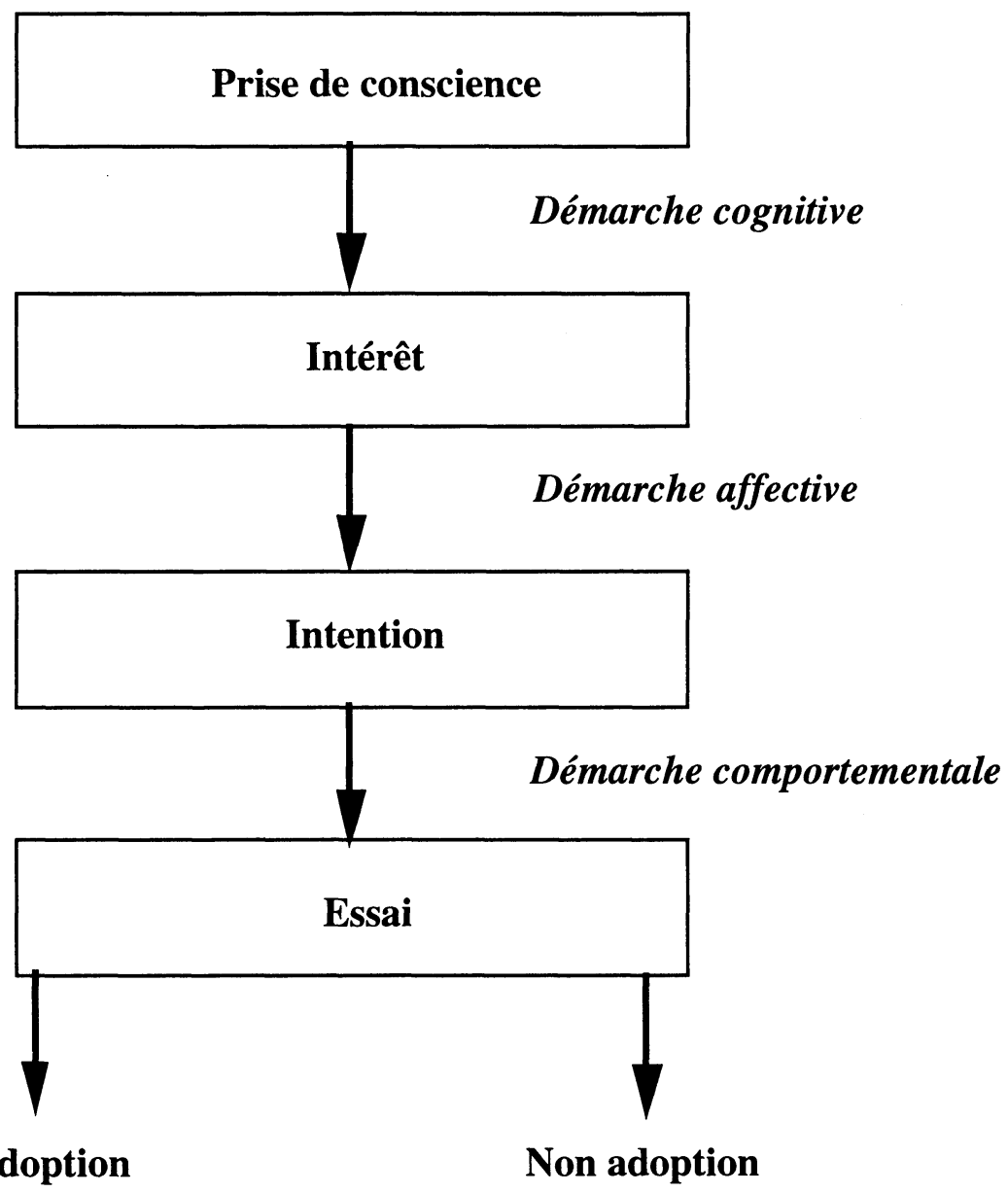


Outre la simple présentation des différentes étapes du processus de décision, certains auteurs ont travaillé sur l'intensité des relations entre ces différentes phases. C'est le cas notamment de l'étude menée par Lim, Sharkey et Kim (1991). D'un point de vue statistique, les liaisons causales entre «perception de l'opportunité » et « intérêt», «intérêt» et «intention», «intention » et «adoption» sont toutes significatives. Il existe cependant des différences d'intensité. La liaison la plus forte est celle qui existe entre «intérêt » et «intention».

La faiblesse de l'intensité de la relation entre «perception de l'opportunité » et «intérêt»s'explique par l'existence de barrières psychologiques. À ce stade, les décideurs des petites firmes hésitent à porter un intérêt à l'exportation, car ils pensent qu'une telle stratégie est réservée aux grandes sociétés. L'intensité relativement faible de la relation entre «intention » et «adoption » est importante à souligner, car la PME est prête à se lancer dans une activité d'exportation. Deux phénomènes peuvent expliquer l'hésitation du dirigeant :

- il a conscience (après une étude des capacités de son entreprise) de son manque d'avantages compétitifs ;

- il perçoit la difficulté de la mise en œuvre (et donc de la réalisation) d'une stratégie d'exportation.

L'analyse des stimuli, des variables exogènes et des différentes phases du processus de décision à l'exportation nous conduit à la présentation des implications managériales qui en découlent.

\section{Les implications managériales}

Bilkey et Tesar (1977) se sont longuement penchés sur les implications en termes de management dans leur étude sur le comportement exportateur des PME/PMI. Ils formulent les recommandations suivantes à l'égard du chef d'entreprise.

- Il doit formuler clairement une stratégie d'exportation. En effet, ils ont relevé une corrélation positive entre la réponse à une commande non sollicitée, d'une part, et entre la qualité du management et la capacité à proposer le produit désiré, d'autre part. Ainsi, un dirigeant répondra d'autant plus rapidement (et avec des chances de succès) à une commande étrangère qu'il aura clairement formulé une stratégie d'exportation.

- Il doit recueillir de nombreuses informations concernant les barrières à l'exportation auxquelles il risque d'être exposé. Le moment venu, lorsqu'il s'engagera dans sa stratégie d'exportation, sa démarche sera facilitée. 
- Il doit avancer de façon graduelle dans son engagement à l'exportation. L'entreprise peut commencer à exporter sur une base expérimentale dans un pays relativement proche (pour la société, à ce stade, les facteurs déterminants sont la qualité et le dynamisme de l'équipe de direction, l'obtention d'une commande non sollicitée). Dans un second temps, l'entreprise, par son expérience, prend une position de force sur ce marché (elle a une bonne vision des risques encourus ainsi que des obstacles à l'entrée sur les marchés étrangers). Enfin, et seulement alors, l'entreprise explore la possibilité d'exporter vers d'autres pays psychologiquement moins proches.

Ces deux auteurs concluent leur étude en indiquant que les PME/PMI ont la possibilité de mener à bien une stratégie d'exportation, cette dernière n'étant pas l'apanage des grandes sociétés. De leur côté, Lim, Sharkey et Kim (1991) mettent en avant le rôle primordial des pouvoirs publics. L'objectif de ces derniers est d'intervenir auprès des PME non conscientes des bénéfices qu'elles peuvent tirer de l'exportation pour développer leur intérêt envers les activités internationales (par rapport à leur modèle, l'objectif sous-jacent est d'améliorer la liaison "perception de l'opportunité-intérêt »). Après avoir développé l'intérêt des sociétés, les pouvoirs publics devraient tenter de créer des occasions de développement international pour les PME afin de faciliter le passage de l'intention à l'adoption.

Pour Olson et Wiedersheim-Paul (1978), le rôle des pouvoirs publics est de jouer sur le style cognitif du dirigeant : ils doivent orienter les entreprises dans leur recherche et leur traitement de l'information. Ortiz-Buonafina (1990) rejoint ces deux auteurs en soulignant que les entreprises ont non seulement besoin d'informations appropriées, mais aussi d'une aide dans leur capacité à analyser et à traiter l'information.

Mescon et Vozikis (1985) distinguent trois stades dans le développement à l'exportation :

Stade 1: la firme ne s'intéresse pas à la possibilité d'exporter régulièrement et/ou elle répond à des offres étrangères ;

Stade 2: la firme étudie la possibilité d'exporter régulièrement et/ou elle exporte sur un ou quelques marchés;

Stade 3: la firme est expérimentée en matière d'exportation sur quelques marchés et elle prospecte de nouveaux marchés.

Selon ces auteurs, l'aide des pouvoirs publics doit être différenciée en fonction du stade auquel se situe l'entreprise (cela est confirmé par l'étude de Cavusgil, 1980). En d'autres termes, le soutien doit être ciblé et qualitatif, et non simplement financier sans différenciation des cas en présence. 


\begin{tabular}{|c|c|c|c|c|c|c|c|c|c|c|c|}
\hline & \begin{tabular}{|l|} 
PAVORD \\
BOGART \\
1975
\end{tabular} & \begin{tabular}{|l|} 
BLKEYY \\
BTESAR \\
1977
\end{tabular} & \begin{tabular}{|l|} 
WIEDERSHEIM \\
PAUL \\
OLSON \\
WELCH \\
1978
\end{tabular} & \begin{tabular}{|l|} 
OLSON \\
WIEDERSEIM \\
PAUL \\
i978 \\
\end{tabular} & MeCONNEL & $\begin{array}{l}\text { Roux } \\
\text { 1979 }\end{array}$ & \begin{tabular}{|l|} 
STEINMANN \\
KUMAR \\
WASNER \\
1980
\end{tabular} & \begin{tabular}{|l|} 
CAVUSGG \\
NEVYN \\
1981
\end{tabular} & \begin{tabular}{|l|} 
REDP \\
1981
\end{tabular} & \begin{tabular}{|l|} 
GARNIER \\
1982
\end{tabular} & \begin{tabular}{|l} 
WELCH \\
1983
\end{tabular} \\
\hline Prise de conscience & $*$ & $*$ & $*$ & $*$ & $*$ & $*$ & $*$ & $*$ & $*$ & $*$ & $*$ \\
\hline \begin{tabular}{|l|} 
Interét pour \\
l'exportation
\end{tabular} & $*$ & * & + & + & + & $*$ & $*$ & $*$ & $*$ & * & \\
\hline $\begin{array}{l}\text { Recherche } \\
\text { d'information }\end{array}$ & + & + & $*$ & $*$ & $*$ & + & & $*$ & + & $*$ & $*$ \\
\hline Évaluation & * & $*$ & + & * & * & * & + & & $*$ & $*$ & + \\
\hline Essai & & $*$ & & & * & * & & $*$ & $*$ & + & $*$ \\
\hline Décision & * & $*$ & $*$ & $*$ & $*$ & $*$ & * & $*$ & $*$ & $*$ & $*$ \\
\hline $\begin{array}{l}\begin{array}{l}\text { Attitude } \\
\text { manageriale }\end{array} \\
\end{array}$ & * & * & * & * & & * & * & $*$ & $*$ & & \\
\hline
\end{tabular}

\begin{tabular}{|c|c|c|c|c|c|c|c|c|c|c|c|}
\hline & \begin{tabular}{|l|} 
DiTCH \\
atal \\
1984
\end{tabular} & \begin{tabular}{|l|} 
MESCON \\
YoZZKIIS \\
1985
\end{tabular} & \begin{tabular}{|l|} 
SHARMA \\
1988
\end{tabular} & $\begin{array}{l}\text { AAFE } \\
\text { NEBENAHL } \\
\text { PASTERAK } \\
\text { 1988 }\end{array}$ & $\begin{array}{l}\text { AXINN } \\
\mathbf{1 9 8 9}\end{array}$ & \begin{tabular}{|l|} 
HIGLER \\
HOOVER \\
1989
\end{tabular} & \begin{tabular}{|l|} 
ORTIZ \\
BLOONFINA \\
1990
\end{tabular} & $\begin{array}{l}\text { LiM } \\
\text { SNARKEY } \\
\text { KMMM } \\
1991\end{array}$ & \begin{tabular}{|l|} 
MiNOR \\
WU \\
COOI \\
1991
\end{tabular} & \begin{tabular}{|l|} 
RAO \\
NAMU \\
1992
\end{tabular} & $\begin{array}{l}\text { CZINKOTA } \\
\text { KONKAINE: } \\
\text { I993 }\end{array}$ \\
\hline Prise de conscience & * & * & * & * & * & * & * & * & * & $*$ & * \\
\hline $\begin{array}{l}\text { Intérêt pour } \\
\text { l'exportation }\end{array}$ & & & & $*$ & $*$ & * & * & * & * & * & $*$ \\
\hline $\begin{array}{l}\text { Recherche } \\
\text { d'information }\end{array}$ & * & $*$ & & + & $*$ & & & * & + & & * \\
\hline Évaluation & $*$ & & & & $*$ & & & & + & & * \\
\hline Essai & * & * & + & & $*$ & & $*$ & & & & * \\
\hline Décision & $*$ & * & $*$ & * & * & * & $*$ & * & * & $*$ & * \\
\hline $\begin{array}{l}\text { Attitude } \\
\text { manageriale }\end{array}$ & & & * & & $*$ & * & * & & * & * & * \\
\hline
\end{tabular}

* Indique que la phase est formalisée dans le modele correspondan

+ Indique que la phase n'est pas formalisée. Elle est cependant intégrée à une autre phase ou sous-entendue par l'auteur.

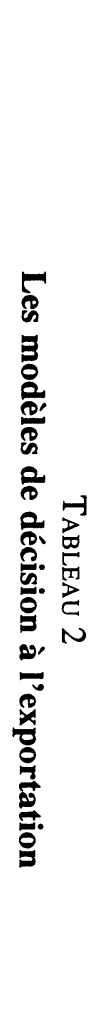


Pour Axinn (1989), les implications managériales sont essentiellement liées à l'orientation internationale du dirigeant. Il doit, à travers la participation à des colloques internationaux et à des «missions export», se sensibiliser aux affaires internationales. Par ce biais, il jugera l'exportation moins complexe, son aversion pour le risque sera moins forte et il mesurera à sa juste valeur l'intérêt de s'engager sur des marchés étrangers. L'aversion pour le risque suscite également l'intérêt de Jaffe, Nebenzahl et Pasternak (1988). Ces derniers mettent en lumière le rôle déterminant que peuvent jouer les pouvoirs publics lors des premiers stades de l'engagement à l'international. Grâce à une panoplie d'outils (assurance, aide financière, informations techniques...), les instances publiques influent directement sur la perception du risque lié à l'exportation.

\section{Conclusion}

On peut considérer que les recherches effectuées ces vingt dernières années sur les modèles intégrés de la décision d'exporter ont déterminé un cadre d'analyse standard caractérisé par différents éléments tels les stimuli, les variables exogènes, le processus interne, le processus de décision et, enfin, le comportement managérial.

Au-delà de ce cadre séquentiel de décision, on remarque que, la plupart du temps, l'engagement des PME à l'international est progressif. En outre, que ce soit lors de la prise de décision ou lors de l'engagement à l'exportation en lui-même, le dirigeant joue incontestablement un rôle déterminant.

En ce qui concerne les recherches à venir, plusieurs auteurs s'accordent pour dire qu'une étude longitudinale permettrait de valider (et d'expliquer) l'enchaînement des phases du processus de décision et des étapes de l'engagement à l'exportation.

Par ailleurs, certains auteurs (Reid, 1981, ainsi que Higler et Hoover, 1989) pensent que les recherches devraient s'effectuer dans un cadre où des éléments comme la taille de l'entreprise, son secteur d'appartenance et sa position géographique seraient contrôlés. Il serait alors plus aisé d'étudier le processus de décision et le rôle du dirigeant au sein de ce processus; de dresser une typologie des comportements à l'exportation. 


\section{Bibliographie}

AABY, N.E. et S.F. SLATER (1989), «Management influences on export performance : a review of empirical literature 1978-8 », International Marketing Review, vol. 6, $\mathrm{n}^{\circ} 4$, p. 7-26.

AXINN, C.N. (1989), «Export performance: do managerial perceptions make a difference », International Marketing Review, vol. 5, $\mathrm{n}^{\circ} 2$, p. 62-71.

BILKEY, J.W. et G. TESAR (1977), «The export behavior of smaller sized Wisconsin manufacturing firms », Journal of International Business Studies, vol. 8, p. 93-98.

BILKEY, J.W. (1978), «An attempted integration of the literature on the export behavior of firms », Journal of International Business Studies, été, p. 33-46.

BeAL, G.M. et J.M. BoHLEN (1957), "The diffusion process ", Iowa Agricultural Extension Service Report, vol. 18.

CAVusGiL, S.T. (1980), «On the internationalisation process of firms», European Research, novembre, p. 273-281.

Cavusgil, S.T. et J.R. Nevin (1981), «Internal determinants of export marketing behavior : an empirical investigation », Journal of Marketing Research, vol. 18, $\mathrm{n}^{\circ} 1$, p. $114-119$.

Colemean, J. (1957), "The diffusion of an innovation», Sociometry, vol. 20, p. $253-270$.

CZInkota, M.R. et I.A. RonkAinen (1993), International Marketing, New York, The Dryden Press, p. 258-267.

Dichtl, E., H.G. GölgMAYR , S. MülleR et M. LeIBOLD (1984), «The export-decision of small and medium-sized firms : a review », Management International Review, vol. $24, n^{\circ} 2$, p. $49-60$.

EMERY, F.E. (1962), Information Decision and Action: A Study of the Psychological Determinants of Change in Farming Techniques, New York, Cambridge University Press.

GARNIER, G. (1982), «Comparative export behavior of small canadian firms in the printing and electrical indusries », dans M. Czinkota et G. Tesar, Export Management: An International Context, New York, Praeger Publishers, p. 113-131.

HASSINGER, E. (1959), «Stage in the adoption process », Rural Sociology, vol. 24, p. 52-53.

Higler, M.T. et R.J. Hoover (1989), «Dynamics of the export decision process », Journal of Global Marketing, vol. 2, n 3, p. 71-93. 
JAFFE, E.D., I.D. NEBENZHAl et H. PASTERNAK (1988), «The export behavior of small and medium-sized israeli manufacturers », Journal of Global Marketing, vol. 2, $n^{\circ} 2$, p. $27-49$.

JOHANSON, J. et J. VAHLNE (1977), «The internationalisation of the firms : four swedish case studies », Journal of International Business Studies, printemps-été, p. 23-32.

KENG, K.A. et T.S. JIUAN (1989), «Differences between small and medium sized exporting and non-exporting firms : nature or nurture », International Marketing Review, vol. 6, n 4 , p. 27-39.

KоH, A.C. (1991), «Relationships among organisational characteristics, marketing strategy and export performance », International Marketing Review, vol. 8, $\mathrm{n}^{\circ} 3$, p. $46-60$.

LAVIDGE, R.J. et G.A. Steiner (1961), «A model of predicting measurements of advertising effectiveness », Journal of Marketing, vol. 25, p. 59-62.

LEE, W.Y. et J.J. BRASH (1978), «The adoption of export as an innovative strategy », Journal of International Business Studies, vol. 9, $\mathrm{n}^{\circ}$ 1, p. 85-93.

KIM, J.S., T.M. SHARKEY et K.I. KIM (1991), «An empirical test of an export adoption model », Management International Review, vol. 31, $\mathrm{n}^{\circ}$ 1, p. 51-62.

MADSEN, T.K. (1989), «Successful export marketing management: some empirical evidence », International Marketing Review, vol. 6, $\mathrm{n}^{\circ}$ 4, p. 41-57.

MCCONNELL, J.E. (1979), «The export decision: an empirical study of firm behavior », Economic Geography, vol. 55, $\mathrm{n}^{\circ}$ 3, p 171-183.

MESCON, T.S. et G.S. VozIKIS (1985) «Small exporters and stages of development: an empirical study», American Journal of Small Business, vol. 40, n 1, p. 49-64.

MieSENBOCK, K.J. (1988), «Small business and exporting : a literature review», International Small Business Journal, vol. 6, $\mathrm{n}^{\circ} 2$, p. 42-61.

MINOR, M., X.Y. WU et M.K. CHOI (1991), «A proposition-based approach to international entry strategy contingencies », Journal of Global Marketing, vol. 4, n ${ }^{\circ} 3$, p. 69-87.

MOON, J. et H. LEE (1990), «On the internal correlates of export stage development: an empirical investigation in the Korean electronics industry », International Marketing Review, vol. 7, $\mathrm{n}^{\circ}$ 5, p. 16-26.

Olson, H.C. et F. Wiedersheim-PAul (1978), «Factors affecting the pre-exportbehavior of non-exporting firms ", dans M. Ghertman et J. Leontiades, European Research in International Business, New York, p. 283-305.

ORTIZ-BuONAFINA, M. (1990), «Small business exporting», Journal of Global Marketing, vol. 3, $\mathrm{n}^{\circ} 4$, p. 33-57. 
PAVORD, W.C. et R. Bogard (1975), «The dynamics of decision to export», Akron Business and Économic Review, vol. 6, printemps, p. 6-11.

RAO, T.R. et G.M. NAIDU (1992), «Are the stages of internationalisation empirically supportable?», Journal of Global Marketing, vol. 6, $\mathrm{n}^{\text {os }} 1-2$, p. 147-170.

REID, S.D. (1981), «The decision maker and export entry and expansion », Journal of International Business Studies, vol. 12, p. 101-112.

Rogers, E.M. (1962), Diffusion of Innovation, New York, The Free Press Glencoe.

Rogers, E.M. et M.D. Yost (1960), «Communication behavior of county extension agent », Ohio Experiment Station Research Bulletin, $\mathrm{n}^{\circ} 850$.

Roux, E. (1979), «The export behavior of small and medium-sized french firms: the role of the manager's profile: some preliminary findings », dans L.G. Mattson et F. Wiedersheim-Paul, Recent Research of the Internationalization of Business, Stockholm, Almquist and Wiskel International, p. 86-101.

Roux, E. (1986), «Les modèles intégrés de la décision d'exporter en PME/PMI», Recherches et applications en marketing, $\mathrm{n}^{\circ} 3$, p. 27-42.

Roux, E. (1991), «Les facteurs explicatifs de la décision d'exporter en PMI : rôle de l'attitude du dirigeant envers le risque », Thèse de doctorat d'État ès sciences de gestion, IAE, Aix-Marseille.

SHARMA, D.D. (1988), «Overseas market entry strategy: the technical consultancy firms ", Journal of Global Marketing, vol. 2, n 2, p. 89-110.

SIMMONDS, K. et H. SMITH (1968), «The first export order : a marketing innovation », British Journal of Marketing, été, p. 93-100.

SimpSon, C.L. et D. KuJAWA (1974), «The export decision process : an empirical inquiry », Journal of International Business Studies, vol. 5, n 1, p. 107-117.

Steinmann, H., B. Kumar et A. WASNer (1980), "Conceptualizing the internationalization process of medium-sized firms. Some preliminary considerations for a research design», Management International Review, vol. 20, $\mathrm{n}^{\circ} 1$, p. 51-66.

WELCH, S.L. (1983), «Managerial decision making : the case of export involvement», Scandinavian Journal of Materials Administration, vol. 9, $\mathrm{n}^{\circ}$ 2, p. 42-47.

Wiedersheim-Paul, F., H.C. Olson et S.L. Welch (1978), «Pre-export activity : the first steps in internationalization », Journal of International Business Studies, vol. $9, \mathrm{n}^{\circ} 1, \mathrm{p} .47-58$. 